\title{
Effet de différentes doses de bouse de vache sur le rendement de la laitue (Lactuca sativa L.) à Tchatchou au Bénin
}

\author{
Vincent Isidore TCHABI", David AZOCLI et Gilbert D. BIAOU

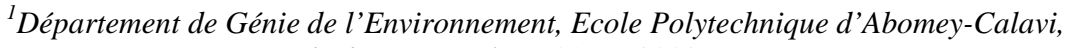 \\ Université d'Abomey -Calavi, 01 BP 2009 Cotonou, Bénin. \\ *Auteur correspondant, E-mail : visidor55@yahoo.fr; Tél :0022995957553
}

\section{RESUME}

Au Bénin, la restauration de la fertilité des sols est une préoccupation pour les agriculteurs qui se heurtent au coût élevé des intrants chimiques. A la recherche d'alternatives peu onéreuses, la présente étude conduite à Tchatchou au Nord-Est du Bénin vise la promotion de l'agriculture biologique à travers la fertilisation organique des sols par la bouse de vache. L'expérimentation a consisté à appliquer différentes doses de bouse de vache pour tester les rendements de la culture de laitue (Lactuca sativa L.). Il ressort des résultats obtenus que la bouse de vache peut assurer un bon développement de la culture de laitue : en effet, le nombre de feuilles par plant a significativement varié en fonction des différentes doses appliquées. Les fortes productions en feuilles par plant, soit 1,75 $\pm 0,64 \mathrm{~g}$ et 1,50 $\pm 0,43 \mathrm{~g}$ ont été obtenues avec des doses de $30 \mathrm{t} / \mathrm{ha}$ et $40 \mathrm{t} / \mathrm{ha}$ respectivement. Le rendement en feuilles le plus élevé, soit 13,35 t/ha $\pm 0,92$ est obtenu avec la dose de $30 \mathrm{t} / \mathrm{ha}$, et la dose optimale pour obtenir un rendement maximal est de 34,8 t/ha.

(C) 2012 International Formulae Group. All rights reserved.

Mots clés: Agriculture biologique, fertilisation organique, Lactuca sativa L., Bénin.

\section{INTRODUCTION}

Au Bénin, pays dont les sols sont en majorité du type ferrugineux, les agriculteurs ploient sous le coût élevé des intrants chimiques pour maintenir leur fertilité (Batiano et Mokwunye, 1991 ; B.E.P.E.A., 1996). Dans ce contexte, l'agriculture biologique offre de nouvelles perspectives avec l'utilisation de composts à base de fiente de volaille, de déjections d'ovins et de guano. En effet, une pratique agricole qui implique l'apport conséquent de substances organiques tels que les résidus de récolte ou différents types de composts, pourrait améliorer la fertilité des sols (Weber et al., 2007) ; et il est connu que l'utilisation des composts produits par les déchets organiques améliore leurs propriétés, rend disponibles les nutriments et réduit les risques de pollution (Douglas et al., 2003), améliorent la structure des sols, augmentent la capacité de rétention en eau et en nutriments, stimulent l'activité microbienne et augmentent les rendements (Kowaljow et Mazzarino, 2007). Toutefois, leur utilisation à l'état brut présente des risques pour la plante et pour l'environnement. Or, la viabilité d'une terre maraîchère dépend avant tout de sa richesse en humus (Tognetti et al., 2008), ce qui fait de l'utilisation des composts une pratique 
courante sur les sols destinés aux cultures maraîchères et constitue une valorisation des sous-produits de l'élevage fortement vulgarisés par l'agriculture biologique (Reganold et al., 2001; Amoussou, 2011).

$\mathrm{Au}$ Nord-Bénin, la bouse de vache est la forme d'intrant organique la plus utilisée, mais son impact sur la culture maraîchère et spécifiquement sur le rendement de la laitue reste à évaluer, but de la présente étude.

\section{MATERIEL ET METHODES \\ Milieu d'étude}

La Commune de Tchaourou, dont fait partie le village de Tchatchou, est comprise entre $8^{\circ} 45^{\prime}$ et $9^{\circ} 20^{\prime}$ de latitude Nord et $2^{\circ} 10^{\prime}$ et $3^{\circ} 40$ de longitude Est. Elle couvre une superficie de $7256 \mathrm{~km}^{2}$, ce qui correspond à $28 \%$ de la superficie du département du Borgou et $6,5 \%$ du territoire national. D'après les résultats du dernier recensement général de la population et de l'habitat de février 2002, la Commune de Tchaourou compte 106852 habitants dont $49,8 \%$ d'hommes et $50,2 \%$ de femmes. Les principaux groupes socioculturels sont les Baribas (34,2\%), les Peulhs $(18,9 \%)$ et les Nagots $(15,8 \%)$ qui s'adonnent principalement à l'agriculture et à l'élevage.

Le climat est du type soudano-guinéen avec deux saisons : une saison pluvieuse s'étendant d'avril à octobre et une saison sèche allant de mi-octobre à mi-avril. L'humidité maximale annuelle est de 97\%, enregistrée en septembre, et un minimum de $20 \%$ enregistré entre janvier et février. Le maximum de température, $32,4{ }^{\circ} \mathrm{C}$, s'observe en mars avec un minimum de $25{ }^{\circ} \mathrm{C}$ en décembre. L'évaporation annuelle maximale est de 245,5 $\mathrm{mm}$ en mars et l'évaporation minimale est de 103,3 mm en août. La hauteur des précipitations varie de $1100 \mathrm{~mm}$ à 1200 mm pour 75 à 140 jours effectifs de pluie.

Le relief est une pénéplaine cristalline faite d'une succession de croûtes surbaissées poly-convexes, constituées de roches cristallines, en majorité métamorphiques. Les sols sont surtout de type ferrugineux tropical.

Le réseau hydrographique est constitué essentiellement du fleuve Ouémé, avec comme affluents l'Okpara et la Térou, qui jouent un rôle écologique important au Bénin.

\section{Matériel végétal}

Le matériel végétal utilisé est la laitue (Lactuca sativa L.), plante annuelle appartenant à la famille des Astéracées, largement cultivée pour ses feuilles tendres consommées comme légume, généralement crues en salade. Le nom « laitue » dérive du latin lactuca, qui rappelle la présence dans cette plante d'un latex blanc caractéristique.

\section{Protocole expérimental}

Le dispositif expérimental est un Bloc Aléatoire Complet $(\mathrm{BAC})$ à 3 répétitions (Figure 1) avec la dose d'engrais organique comme le seul facteur à 4 modalités (4 doses) : $0, \quad 20, \quad 30$ et 40 t/ha (notées respectivement $\mathrm{T} 0, \mathrm{~T} 20, \mathrm{~T} 30$ et $\mathrm{T} 40$ ), ce qui correspond à des quantités respectives de 0 ; 4,8; 7,2 et 9,6 kg par parcelle élémentaire (ou planche) dont les dimensions sont de $2 \mathrm{~m} \mathrm{x}$ $1,2 \mathrm{~m}$; soit au total 4 doses $\mathrm{x} 3$ répétitions, ce qui correspond à 12 parcelles élémentaires. Des allées de 0,5 m ont été observées entre les parcelles élémentaires et $1 \mathrm{~m}$ entre les blocs.

La pépinière a été mise en place le 08 mai 2011. Trois semaines avant le repiquage (le 15 mai 2011), les bouses de vache ont été enfouies dans le sol sur chacune des parcelles élémentaires sous forme de fumure de fond et en dose unique. Le repiquage a eu lieu le 10 juin 2011 et a été effectué suivant les écartements de $20 \mathrm{~cm}$ x $20 \mathrm{~cm}$, soit une densité de semis de 187500 plants par hectare. Un désherbage manuel et à la houe est effectué chaque semaine pour réduire l'effet des mauvaises herbes sur la croissance et la production des plantes. L'arrosage se fait deux fois par jour soit deux arrosoirs de 11 litres par planche. 


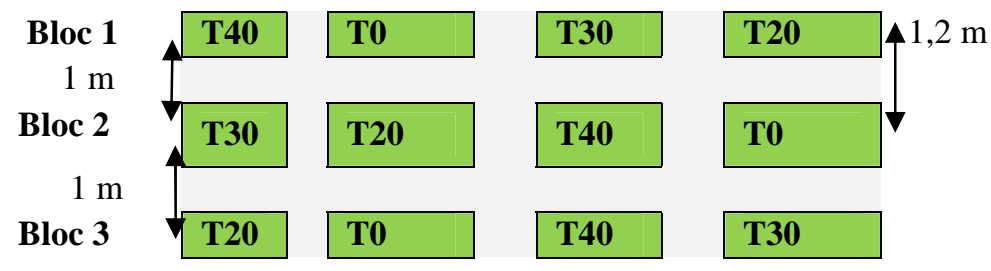

Figure 1: Schéma du dispositif expérimental avec la répartition des traitements.

\section{Variables et paramètres mesurés}

- Nombre de plants récoltés: il s'est agi de compter tous les plants sur une surface de 0,6 $\mathrm{m}$ x 0,6 m prise dans les lignes centrales.

- Nombre de feuilles sur les plants à maturité, en comptant toutes les feuilles sur 10 plants sélectionnés au hasard.

- Poids frais des feuilles : cette observation a consisté à peser à l'aide d'une balance de $4 \mathrm{~kg}$ de portée, de marque ACCULAB Sartorius, les feuilles de tous les plants récoltés sur une surface de 0,60 x 0,60 m, tout en éliminant les plants et lignes de bordure.

\section{Analyses statistiques}

Le logiciel Excel a été utilisé pour la saisie des données. Les différentes données à savoir le nombre de plants récoltés par planche échantillonnée, le nombre de feuilles par plant à maturité en comptant toutes les feuilles sur 10 plants sélectionnés au hasard, le poids frais des feuilles, dont les moyennes et les écarts-types sont soumises à un rapport de variance inter et intra-groupe ANOVA.

Les analyses statistiques ont été faites à l'aide du logiciel SPSS v16.0. Le test de StudentNewman-Keuls de Dagnelie (1986) au seuil de $5 \%$ est utilisé pour faire la comparaison deux à deux des moyennes.

\section{RESULTATS}

Effet de différentes doses d'application de bouses de vache sur le nombre de feuilles

Les bouses de vache ont des effets très hautement significatifs $(\mathrm{P}<0,001)$ sur le nombre de feuilles de laitue à la récolte (Tableau 1). Le Tableau 1 indique par ailleurs que les doses de 30 t/ha et 40 t/ha ont présenté le plus grand nombre de feuilles $(17,79 \pm 2,35$ et $19,62 \pm 3,86$ respectivement $)$, comparativement aux autres doses $(0 \mathrm{t} / \mathrm{ha}$ et $20 \mathrm{t} / \mathrm{ha}$ ) dont les effets sur le nombre de feuilles sont significativement différents au seuil de $5 \%$. La dose minimum pour observer un début d'effet est de 20 t/ha.

Effet de différentes doses d'application de bouses de vache sur la productivité en feuilles

La Figure 2 illustre la productivité en feuilles en fonction des différentes doses appliquées. Il en ressort que les fortes productions en feuilles par plant sont respectivement de $1,75 \mathrm{~g} \pm 0,64 \mathrm{~g}$ et $1,50 \mathrm{~g} \pm$ $0,43 \mathrm{~g}$ pour les doses de $30 \mathrm{t} / \mathrm{ha}$ et $40 \mathrm{t} / \mathrm{ha}$ durant la période de l'étude. Le fait que les productions obtenues avec ces deux doses ne soient pas significativement différentes au seuil de 5\% montre, ici aussi, que la dose optimale serait de 30 t/ha. Cette Figure indique par ailleurs que les productions obtenues avec les doses 0 t/ha et 20 t/ha ne sont pas significativement différentes au seuil de $5 \%$ pour ce paramètre.

En ce qui concerne le rendement en feuilles (Tableau 2), on remarque aussi que la plus forte valeur s'observe avec la dose de 30 t/ha, et qu'aucune différence au seuil de $5 \%$ ne s'observe entre les doses 0 et 20 t/ha d'une part, et les doses 30 et 40 t/ha d'autre part. 


\section{Détermination de la dose optimale}

La courbe ascendante (Figure 3) présente la surface de réponse du rendement en feuilles de la laitue soumise à différentes doses d'application de bouses de vache. Elle a été obtenue par l'équation: $Y=-0,005 x^{2}+$
$0,348 x+6,782\left(R^{2}=0,851\right)$ avec $Y=$ Rendement et $\boldsymbol{x}=$ Doses d'application. La dose optimale qui en découle est de $35 \mathrm{t} / \mathrm{ha}$, avec $85,1 \%$ de l'évolution du rendement expliqués par les différentes doses.

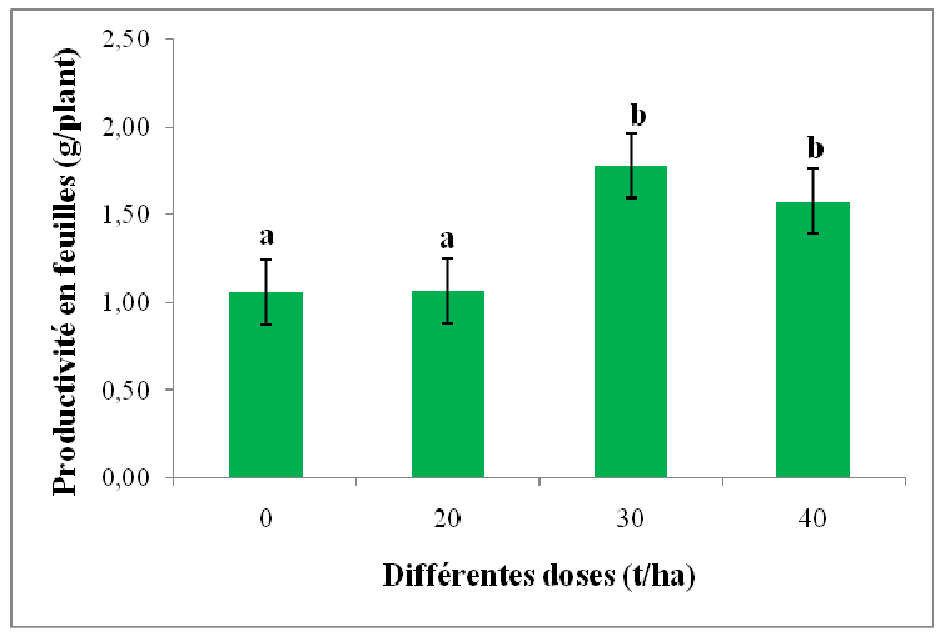

Figure 2: Effet des trois doses d'application sur la productivité en feuilles fraîches de laitue. Les moyennes suivies des mêmes lettres alphabétiques ne sont pas significativement différentes au seuil de 5\% avec le test de Student Newman - Keuls.

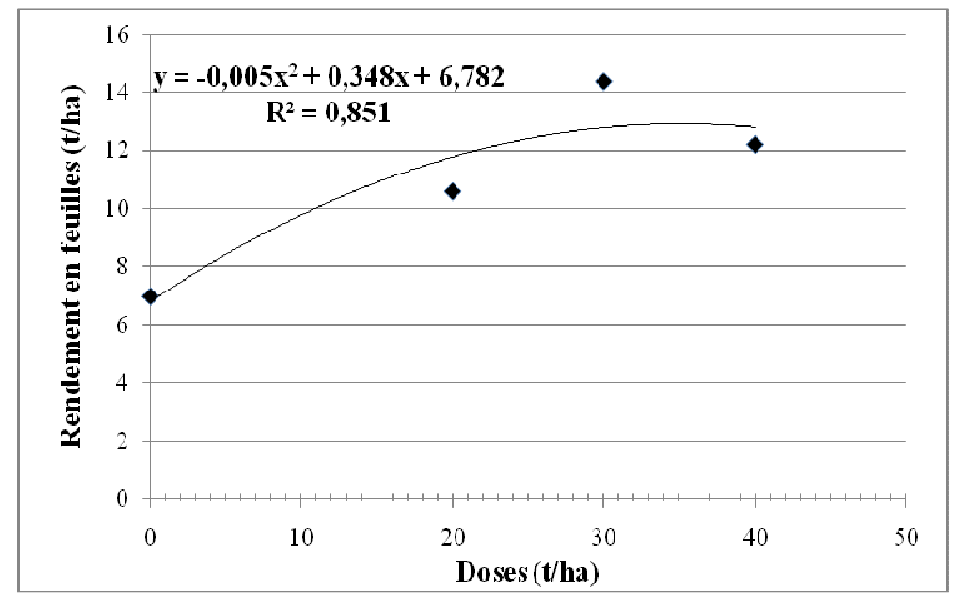

Figure 3: Courbe montrant l'effet de différentes doses d'application de bouses de vache sur le rendement en feuilles de la laitue. 
Tableau 1: Effet de trois doses de bouse de vache (valeurs moyennes \pm écart-type) sur le nombre de feuilles fraîches par plant de laitue.

\begin{tabular}{lcccc}
\hline Doses (t/ha) & Moyennes & Ecart-type & Intervalles & Probabilité \\
\hline $\mathbf{0}$ & $12,33 \mathrm{a}$ & 1,88 & $8-16$ & \\
$\mathbf{2 0}$ & $14,97 \mathrm{~b}$ & 3,17 & $10-22$ & $<0,001$ \\
$\mathbf{3 0}$ & $17,79 \mathrm{c}$ & 2,35 & $13-24$ & \\
$\mathbf{4 0}$ & $19,62 \mathrm{c}$ & 3,86 & $14-32$ & \\
\hline
\end{tabular}

Les moyennes suivies des mêmes lettres alphabétiques ne sont pas significativement différentes au seuil de 5\% avec le test de Student Newman - Keuls.

Tableau 2: Effet de trois doses de bouse de vache (valeurs moyennes \pm écart-type) sur le rendement en feuilles ( $\mathrm{t} / \mathrm{ha})$ de la laitue.

\begin{tabular}{lccc}
\hline Doses (t/ha) & Moyennes & Ecart-type & Probabilité \\
\hline $\mathbf{0}$ & $6,98 \mathrm{a}$ & 0,48 & \\
$\mathbf{2 0}$ & $10,58 \mathrm{a}$ & 1,93 & $<0,05$ \\
$\mathbf{3 0}$ & $13,35 \mathrm{~b}$ & 0,92 & \\
$\mathbf{4 0}$ & $12,19 \mathrm{~b}$ & 4,57 & \\
\hline
\end{tabular}

\section{DISCUSSION}

Par rapport aux différentes doses d'application de bouse sur le nombre de feuilles, il est à noter que les résultats obtenus révèlent qu'il s'agit d'un engrais de qualité, notamment pour sa forte teneur en azote, élément essentiel au développement des végétaux (M.R.E.C.D., 2002). En effet, M.R.E.C.D. (2002) précise que l'azote est un facteur essentiel de croissance des plants, surtout au niveau des feuilles et des tiges. Ces résultats sont en concordance avec ceux obtenus par Biaou (2010), qui a travaillé sur la laitue et la carotte. Le nombre de feuilles obtenu avec les doses de 30 t/ha et de 40 t/ha n'étant pas significativement différent au seuil de $5 \%$, la dose optimale pour obtenir le maximum de feuilles à la récolte serait de 30 t/ha.

En ce qui concerne la redondance statistiquement prouvée des valeurs obtenues avec les doses de 30 t/ha et 40 t/ha relativement à l'effet de différentes doses d'application de bouse sur la productivité en feuilles, elle est bien expliquée par Dapoigny et al. (1997), Tittonel et al. (2003), et Biaou (2010) : cette baisse du rendement à 40 t/ha s'explique par le fait qu'à cette dose, l'excès en azote a été préjudiciable pour les plants, laissant entrevoir que la décomposition de l'azote contenu dans les bouses de vache n'a pas été totale compte tenu de la quantité apportée. Aussi, selon Bressoud et al. (2003), la fertilisation doit-elle être mesurée et limitée pour éviter l'excès d'azote, qui sera finalement inutilisable. A ce sujet, Sou et al. (2007) ont indiqué qu'un apport excessif d'azote peut entraîner une baisse de rendement, due à un développement accru des tiges au détriment des feuilles.

Au sujet de la détermination de la dose optimale, le résultat obtenu est similaire à celui d'Amoussou (2011) qui a obtenu une dose optimale de 36 t/ha sur sols ferrugineux tropicaux avec la variété locale de tomate «Tomati ». On peut donc valider cette équation. 


\section{Conclusion}

Le premier objectif spécifique de cette étude est d'évaluer l'effet de la bouse de vache sur le rendement de la laitue. Il en ressort que la bouse de vache a permis un bon développement de la laitue, à partir d'une dose minimale de 20 t/ha. Le deuxième objectif spécifique est de déterminer la dose optimale, qui permettra d'obtenir de meilleurs rendements. Elle est de 34,8 t/ha. $85,1 \%$ de l'évolution du rendement relèvent des différentes doses appliquées, ce qui est très significatif.

\section{REFERENCES}

AFSSA (Agence Française de Sécurité Sanitaire des Aliments). 2007. Evaluation Nutritionnelle et Sanitaire des Aliments Issus de l'Agriculture Biologique. AFSSA: Paris, France; 164 p.

Amoussou AD. 2011. Effets de deux engrais organiques sur la croissance et le rendement de la tomate (Lycopercicum esculentum Mill) dans les périmètres maraîchers de la commune de Parakou. Mémoire d'Ingénieur Agronome FA/UP, $57 \mathrm{p}$.

ASECNA. 2010. Diagramme climatique de la station synoptique de Parakou pour la période de 1971 - 2010. ASECNA, Bénin.

Batiano A, Mokwunye AU. 1991. Rôle des résidus d'engrais et de récolte en allégeant des contraintes de fertilité du sol à la production végétale en se référant tout particulièrement aux zones sahéliennes et soudaniennes de l'Afrique Occidentale. Fer. Res., 29: 117-125.

BEPEA. 1996. Document préparatoire du Forum National de lancement du processus d'élaboration du programme d'action de lutte contre la désertification. MEHU/GTZ, p. 20-24.

Biaou ODB. 2010. Valorisation de l'agriculture biologique: Effet de différentes sources d'engrais organiques sur le rendement et la qualité de la laitue et de la carotte sur sol ferralitique au Sud Bénin. Mémoire d'Ingénieur Agronome FA/UP, 81p.

Bressoud F, Parès L, Lecompte F. 2003. Tomate d'abri froid. Fertilisation et restriction en azote: le standard actuel inadapté au sol. Réussir Fruits et Légumes, 220 : 30-31.

Dapoigny L, Fleury A, Robin P. 1997. Relation entre la vitesse relative de croissance et la teneur en azote dans la laitue (Lactuca sativa L.). Effets du rayonnement et de la température. Agronomie, 17: 35-41.

Douglas JT, Aitken MN, Smith CA. 2003. Effects of five non-agricultural organic wastes on soil composition and on the yield and nitrogen recovery on Italian ryegrass. Soil Use Man., 19: 135-138.

Golam R, Thapa GB. 2003. Sustainaibility analysis of ecological and conventional agricultural systems in Bangladesh. World Developpement, 31(10): 17211741.

Kowaljow E, Mazzarino MJ. 2007. Soil restoration in semiarid Patagonia: chemical and biological response to different compost quality. Soil Biol. Bioch., 39: 1580-1588.

Lotter DW, Seidel R, Liebhart W. 2003. Exécution des systèmes organiques et conventionnels d'emblavage en année extrême de climat. Journal Américain de l'Agriculture Alternative, 18:1-3.

Mauricourt G. 2005. Agriculture et Santé : l'Impact des Pratiques Agricoles sur la Qualité de vos Aliments. Editions Dangles, Médecine Vétérinaire de Bucarest, Faculté d'Agriculture; 286p.

Ministère des Relations Extérieurs, de la Coopération et du Développement (). 2002. Momento de l'Agronome ( $\left.7^{\mathrm{e}} \mathrm{edn}\right)$. MRECD: Paris; 379 p.

Reganold JP, Glover JD, Andrews PK, Hinman HR. 2001. Organic farming and 
the sustainability of agricultural systems. p. $21-40$.

Robitaille R. 2010. Le point sur la fertilisation en production biologique de la tomate de serre. 16p. http://www.agrireseau.qc.ca/ agriculturebiologique/navigation.aspx?

Sou M, Yacouba H, Mermoud A. 2007. Valorisation du pouvoir fertilisant des eaux usées en agriculture maraîchère. EIER, Ouagadougou, Burkina Fasso, 13 p.

Tittonell PA, De Grazia J, Chiesa A. 2003. Nitrate and dry matter concentration in a leafy lettuce (Lactuca sativa L.) cultivar as affected by fertilization and plant population. Agricultura Tropica, 36: 8287.

Tognetti C, Mazzarino MJ, Laos F. 2008. Compost of municipal organic waste: effects of different management practices on degrability and nutrient release capacity. Soil Biol. Bioch., 49: 22902296.

Weber J, Karczewska A, Drozd J, Lieznar M, Lieznar S, Jamroz E, Kocowiez A. 2007. Agricultural and ecological aspects of sandy soil as affected by the application of municipal solid waste composts. Soil Biol. Bioch., 39: 1294-1302. 